\title{
Simulating digital micromirror devices for patterning coherent excitation light in structured illumination microscopy
}

\author{
Mario Lachetta ${ }^{1,2}$, Hauke Sandmeyer ${ }^{3}$, Alice Sandmeyer ${ }^{1}$, Jan Schulte \\ am Esch $^{2}$, Thomas Huser ${ }^{1}$, Marcel Müller ${ }^{1}$ \\ ${ }^{1}$ Biomolecular Photonics, Faculty of Physics, Bielefeld University, \\ Universitätsstraße 25, 33501 Bielefeld, Germany \\ ${ }^{2}$ Department of General and Visceral Surgery, Protestant Hospital of Bethel Foundation, \\ Schildescher Str. 99, 33611 Bielefeld, Germany \\ ${ }^{3}$ Numerical Simulations and Field Theory, Faculty of Physics, Bielefeld University, \\ Universitätsstraße 25, 33501 Bielefeld, Germany
}

Keywords: SIM, structured illumination microscopy, DMD, digital micromirror device, coherent light simulation, super-resolution optical microscopy

\section{Summary}

Digital micromirror devices (DMDs) are spatial light modulators that employ the electro-mechanical movement of miniaturized mirrors to steer and thus modulate the light reflected of a mirror array. Their wide availability, low cost and high speed make them a popular choice both in consumer electronics such as video projectors, and scientific applications such as microscopy.

High-end fluorescence microscopy systems typically employ laser light sources, which by their nature provide coherent excitation light. In super-resolution microscopy applications that use light modulation, most notably structured illumination microscopy (SIM), the coherent nature of the excitation light becomes a requirement to achieve optimal interference pattern contrast. The universal combination of DMDs and coherent light sources, especially when working with multiple different wavelengths, is unfortunately not straight forward. The substructure of the tilted micromirror array gives rise to a blazed grating, which has to be understood and which must be taken into account when designing a DMD-based illumination system.

Here, we present a set of simulation frameworks that explore the use of DMDs in conjunction with coherent light sources, motivated by their application in SIM, but which are generalizable to other light patterning applications. This framework provides all the tools to explore and compute DMD-based diffraction effects and to simulate possible system alignment configurations computationally, which simplifies the system design process and provides guidance for setting up DMD-based microscopes. 


\section{Introduction}

Spatial light modulators (SLMs) offer a robust and fast way to pattern the excitation light in a fluorescence microscope. This can be employed for various illumination schemes [1], for example to achieve selective (de)activation of photo-switchable dyes [2], and most notably, to achieve background suppression and resolution enhancement in structured illumination microscopy (SIM) [3-8], a widely used, fast super-resolution microscopy technique [9-13]. Typical SLMs are based on liquid crystal technology, and thus operate by electrically modulating the phase (or amplitude) of light through their active material. Digital micromirror devices (DMDs), on the other hand, work electro-mechanically, by individually flipping the orientation of each mirror between 2 pre-defined states. Because of their widespread use in consumer devices such as video projectors, DMDs are available at relatively low cost and in a variety of sizes. They also offer high switching
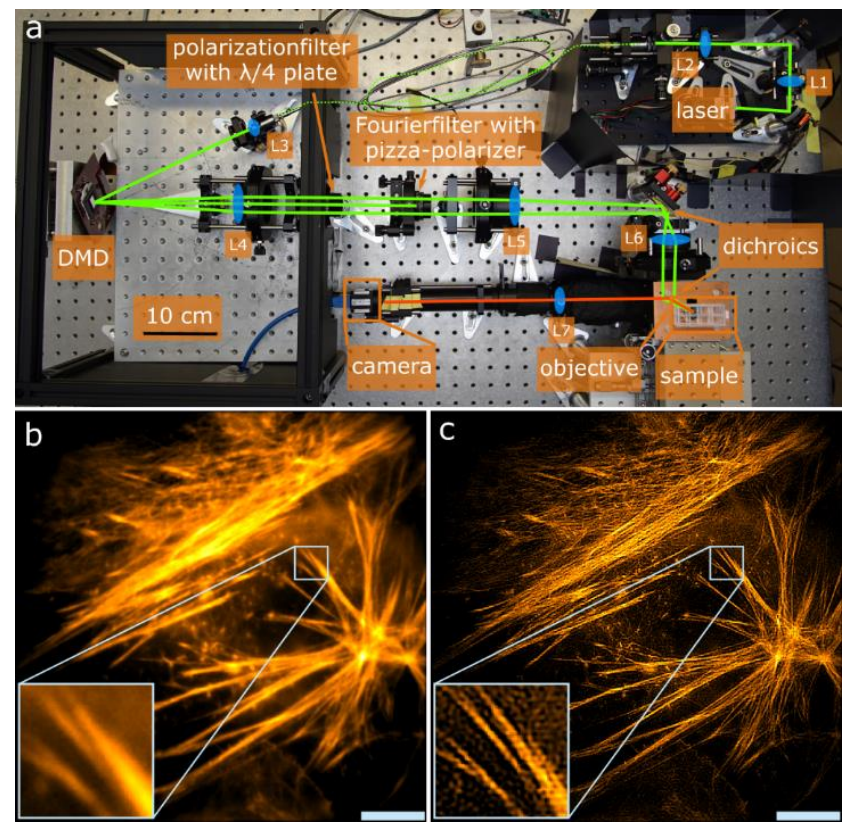

Figure 1: DMD-SIM microscope and measurements

(a) Compact \& cost-effective SIM system based on a DMD with a $532 \mathrm{~nm}$ laser. The design of this microscope necessitated and motivated the research presented in this article. (b) $36 \mu m \times 36 \mu \mathrm{m}$ wide field of fixed U2OS cell labeled with Phalloidin Atto532 acquired using the instrument with $20 \mathrm{~ms}$ exposure per raw frame. The actin filaments are not distinguishable. (c) SIM data of (b). The actin filaments are distinguishable. (scale bar $5 \mu \mathrm{m}$, inset $2.8 \mu \mathrm{m} \times 2.8 \mu \mathrm{m})$

speeds, they can handle high light intensities,

and depending on coating, are not sensitive to light polarization. This makes them an interesting option for many SLM applications in microscopy [14-22]. However, the jagged nature of the micromirror array gives rise to the blazed grating effect that becomes rather annoying and detrimental when using DMDs in combination with a coherent light source $[23,24]$. Thus, if DMDs are selected as active light modulation systems in a fluorescence microscope based on laser light sources, this effect must be well understood and needs to be taken into consideration.

The work presented here was motivated by our wish to expand the range of applications of a DMDbased SIM system (see Figure 1), where a coherent laser source is the primary source for the SIM interference pattern, giving rise to optimal pattern contrast [14]. We have developed a set of simulation frameworks, that allow us to simulate the propagation of coherent light reflected off of a DMD at different angles of incidence, at different wavelengths and with the DMD displaying arbitrary patterns. This facilitates us to explore the feasibility of optical layouts, to determine which simplifications (e.g. keeping optical elements in a single plane on a table) are possible, and to choose the proper wavelength combinations that could be used in a multi-color DMD-based system. While our work is motivated by and centred around SIM, most of the findings should apply to any other, arbitrary DMD-based microscope systems that employ coherent illumination.

\section{Methods}

If DMDs are to be used with coherent illumination, it is essential to know and understand the resulting diffraction patterns generated by both the pattern displayed on the DMD and, much more importantly, the structure of the tilted micromirrors that is native to the device. For this purpose, we have developed three simulation algorithms, each with different assumptions and resulting strengths. Additionally, we have developed an analytical solution that can be used along the diagonal of the DMD. All three 
algorithms are based on the same physical model of propagating electromagnetic fields interacting with the DMD's structure.

\section{General modelling of coherent light and DMDs:}

The general model was derived for the a previously developed DMD-based SIM system [14], and, for completeness, is summarized here, as it forms the basis of our simulation approaches. For incident and diffracted directions we use the normalized vectors $\vec{a}=\left(a_{x}, a_{y}, a_{z}\right)$ and $\vec{b}=\left(b_{x}, b_{y}, b_{z}\right)$ with the angle coordinates $\left(\varphi_{a}, \vartheta_{a}\right)$ and $\left(\varphi_{b}, \vartheta_{b}\right)$ :

$$
\begin{aligned}
& \vec{a}\left(\varphi_{a}, \vartheta_{a}\right)=\left(\begin{array}{c}
a_{z} \cdot \tan \left(\varphi_{a}\right) \\
a_{z} \cdot \tan \left(\vartheta_{a}\right) \\
a_{z}
\end{array}\right) \text { with } \quad a_{z}=\sqrt{\frac{1}{\tan ^{2}\left(\varphi_{a}\right)+\tan ^{2}\left(\vartheta_{a}\right)}} \\
& \vec{b}\left(\varphi_{b}, \vartheta_{b}\right)=\left(\begin{array}{c}
b_{z} \cdot \tan \left(\varphi_{b}\right) \\
b_{z} \cdot \tan \left(\vartheta_{b}\right) \\
b_{z}
\end{array}\right) \text { with } b_{z}=\sqrt{\frac{1}{\tan ^{2}\left(\varphi_{b}\right)+\tan ^{2}\left(\vartheta_{b}\right)}}
\end{aligned}
$$

For every incident direction, all diffracted directions must be considered. Since the DMD is basically a two-dimensional array of mirrors, we start with modelling a single mirror with defined dimensions:

$$
\vec{c}(s, t)=(s, t, 0) \quad \text { with } \quad 0 \leq s \leq w, 0 \leq t \leq w
$$

Here, $w$ is the width and height of the mirror, and $s$ and $t$ are parameters for the $\mathrm{x}$ - and $\mathrm{y}$-directions, respectively. To model the tilt state each single mirror in a DMD array can be rotated by the angle $\gamma$ around the normalized diagonal axis $\vec{n}=\left(n_{x}, n_{y}, n_{z}\right)=\frac{1}{\sqrt{2}}(1,1,0)$ via the rotation matrix:

$$
R_{n}(\gamma)=\left(\begin{array}{ccc}
n_{x}^{2}(1-\cos \gamma)+\cos \gamma & n_{x} n_{y}(1-\cos \gamma)-n_{z} \sin \gamma & n_{x} n_{z}(1-\cos \gamma)+n_{y} \sin \gamma \\
n_{y} n_{x}(1-\cos \gamma)+n_{z} \sin \gamma & n_{y}^{2}(1-\cos \gamma)+\cos \gamma & n_{y} n_{z}(1-\cos \gamma)-n_{x} \sin \gamma \\
n_{z} n_{x}(1-\cos \gamma)-n_{y} \sin \gamma & n_{z} n_{y}(1-\cos \gamma)+n_{x} \sin \gamma & n_{z}^{2}(1-\cos \gamma)+\cos \gamma
\end{array}\right)
$$

To shift a single mirror, we use the native grid $\vec{m}\left(m_{x}, m_{y}\right)=m\left(m_{x}, m_{y}, 0\right)$ with the micromirror pitch $m=w+g$. The gap between the non-tilted mirrors is $g . m_{x} \in \mathbb{N}_{0}$ and $m_{y} \in \mathbb{N}_{0}$ are the indices of the grid points (the single mirrors). This leads us to the following expression to describe each single mirror on the DMD:

$$
\vec{d}_{m_{x}, m_{y}}\left(\gamma_{m_{x}, m_{y}}, s, t, m\right)=R\left(\gamma_{m_{x}, m_{y}}\right) \cdot \vec{c}(s, t)+m\left(m_{x}, m_{y}, 0\right)
$$

We can model monochromatic coherent light using the Fraunhofer / far-field approximation with a time-independent electric field, which depends on the beam profile $I_{0}(\vec{a}, \vec{d})=\left|E_{0}(\vec{a}, \vec{d})\right|^{2}$ projected onto the DMD:

$$
E\left(E_{0}(\vec{a}, \vec{d}), \Phi\right)=E_{0}(\vec{a}, \vec{d}) \cdot e^{i \Phi} \quad \text { with } \quad \Phi=l \cdot \frac{2 \pi}{\lambda}=\vec{d}(\vec{a}-\vec{b}) \frac{2 \pi}{\lambda}
$$

$E_{0}$ represents the incidence amplitude and $\Phi$ the resulting phase, which is dependent on the path length $l=l_{a}+l_{b}=\vec{d} \vec{a}-\vec{d} \vec{b}$ and the wavelength $\lambda$ (Figure 2a). For the electric field diffracted at a specific point on the DMD we obtain:

$$
E_{m_{x}, m_{y}}^{\text {one point }}\left(\vec{a}, \vec{b}, \gamma_{m_{x}, m_{y}}, s, t, m, \lambda\right)=E_{m_{x}, m_{y}}^{0}\left(\vec{a}, \gamma_{m_{x}, m_{y}}, s, t\right) \cdot \exp \left(\frac{2 \pi i}{\lambda} \vec{d}_{m_{x}, m_{y}}\left(\gamma_{m_{x}, m_{y}}, s, t, m\right) \cdot(\vec{a}-\vec{b})\right)
$$

To calculate the diffracted field distribution we need to integrate over each single mirror and calculate the sum over the entire DMD with $N_{x}$ and $N_{y}$ as the number of single mirrors in x- and y-direction:

$$
E_{D M D}\left(\vec{a}, \vec{b}, \gamma_{m_{x}, m_{y}}, w, m, N_{x}, N_{y}, \lambda\right)=\sum_{m_{x}=0, m_{y}=0}^{N_{x}-1, N_{y}-1} \iint_{0}^{w} E_{m_{x}, m_{y}}^{0}\left(\vec{a}, \gamma_{m_{x}, m_{y}} s, t\right) \cdot \exp \left(\frac{2 \pi i}{\lambda} \vec{d}_{m_{x}, m_{y}}\left(\gamma_{m_{x}, m_{y}}, s, t, m\right) \cdot(\vec{a}-\vec{b})\right) d s d t
$$



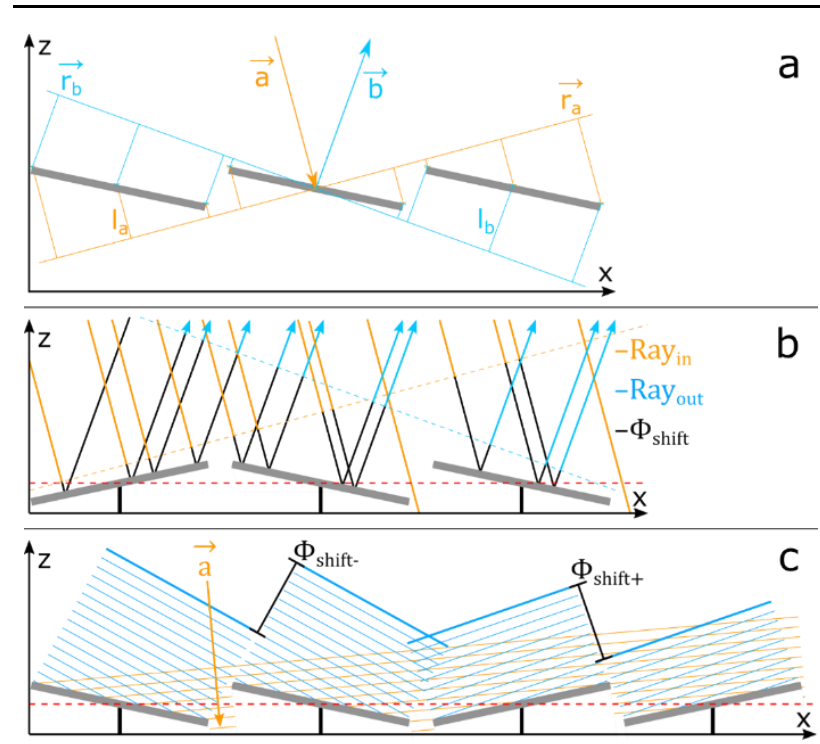

a
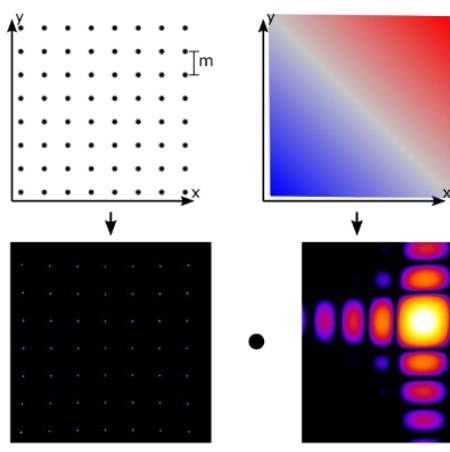

$\downarrow$
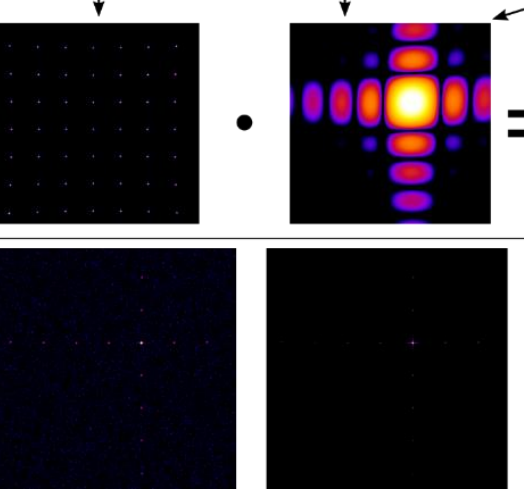

Figure 2: Illustration and comparison of algorithms for the simulation of diffraction with DMDs

Modelling of (coherent) light that is diffracted at a DMD surface. All diffraction images shown are in logarithmic intensity representation and were generated with an array of $50 \times 50$ micromirrors at $532 \mathrm{~nm}$ wavelength. Each micromirror has a size of $7.56 \mu \mathrm{m} \times 7.56 \mu \mathrm{m} . \varphi_{a}=-\vartheta_{\mathrm{a}}=$ $-21^{\circ}$ (blazed condition) was chosen as angle of incidence. The diffraction images are shown for both angles $\varphi_{b}$ and $\vartheta_{b}$ with an angle range of $15^{\circ}$ to $15^{\circ}$. (a) General determination of the phase shift for different points of a planar wave front, which is incident on the DMD in direction $\vec{a}$ and is diffracted in direction $\vec{b}$. This approach is correct in the far-field / Fraunhofer approximation. (b) Ray tracing approach: Modeling of rays and their phase shift, which are incident on the DMD in the direction $\vec{a}$ and diffracted in the direction $\vec{b}$. (c) Analytical phase shift approach: Simplified modelling of diffraction images in the form of wave fronts reflected by micromirrors, where the diffraction image of a single mirror is analytically known. (d) Grating approach: In the upper row the native grating of the DMD array, and a single mirror are shown schematically. In the lower row the corresponding diffraction images and their product, which results in the diffraction image of the entire $D M D$, are shown. (e) Comparison of the diffraction images (from left to right): Ray tracing approach, analytical phase shift approach, grating approach.

This expression depends on many parameters and generally cannot be simplified further analytically. In order to be able to calculate diffraction patterns numerically and in adequate time, it is thus necessary to make further assumptions to simplify the calculation. Depending on how these assumptions are chosen, different approaches emerge capable of modelling different system constraints:

\section{Ray tracing approach:}

We approximate integrating over every single mirror and summing over the DMD (eq. (7)) by running a Monte-Carlo-simulation with rays which will be summed up (Figure 2b). This yields a computationally feasible (if somewhat slow) simulation that does not introduce further constraints or approximations not inherent to the Monte-Carlo process. To simulate a collimated Gaussian incident laser beam, we use a Gaussian probability density for the generation of $K$ randomized incident beams with this equation of a line

$$
\vec{f}_{k}=\vec{h}_{k}+u \vec{a} \quad \text { with } \quad k \in \mathrm{N}_{0}, \quad u \in \mathbb{R} \quad \text { and } \quad k<K
$$

and with $\vec{h}_{k}$ as support vector, $\vec{a}$ as normalized incident direction vector and the parameter $u$. Here, $\vec{h}_{k}$. $\vec{a}=0$ because $\vec{h}_{k}$ and $\vec{a}$ are perpendicular to each other. $\vec{h}_{k}$ is Gaussian randomized for each ray $\vec{f}_{k}$. To obtain the point of diffraction on the DMD we must calculate the intersection point $\vec{p}_{k}=\left(m_{x}, m_{y}, s, t, u\right)$ of $\vec{f}_{k}$ and $\vec{d}$. For each ray we assume that the intensity is $E_{m_{x}, m_{y}}^{0}=1$. This simplifies eq. (6) to:

$$
E_{m_{x}, m_{y}}^{\text {one ray }}\left(\vec{a}, \vec{b}, \vec{p}_{k}, \lambda\right)=E_{\text {in }}\left(\vec{a}, \vec{p}_{k}, \lambda\right) \cdot E_{\text {out }}\left(\vec{b}, \vec{p}_{k}, \lambda\right)=\exp \left(\frac{2 \pi i}{\lambda} \vec{d}\left(\vec{p}_{k}\right) \vec{a}\right) \cdot \exp \left(-\frac{2 \pi i}{\lambda} \vec{d}\left(\vec{p}_{k}\right) \vec{b}\right)
$$

A Gaussian beam profile is already considered by the probability distribution of the rays. The field composed of contributions from all rays needs to be summed up to result in the final ray tracing expression for the diffracted field distribution (Figure 2e, left). This simplifies eq. (7) to: 


$$
E_{D M D}\left(\vec{a}, \vec{b}, \gamma_{m_{x}, m_{y}}, w, m, N_{x}, N_{y}, \lambda\right)=\sum_{k=0}^{K-1} \exp \left(\frac{2 \pi i}{\lambda} \vec{d}\left(\vec{p}_{k}\right)(\vec{a}-\vec{b})\right)
$$

In this ray-tracing approach, both the assumption of a Gaussian beam profile and especially of fully monochromatic (i.e. coherent) light could easily be changed. Thus, by using a different ray distribution, and by allowing for the single rays (eq. 9) to follow a wavelength and phase distribution when being summed up (eq. 10), it would easily be possible to simulate for example an arbitrary profile of a partially coherent source.

\section{Analytic phase shifting approach:}

For this approach we assume that the field amplitude over each single mirror is constant: $E_{m_{x}, m_{y}}^{0}\left(\vec{a}, \gamma_{m_{x}, m_{y}}, s, t\right) \rightarrow E_{m_{x}, m_{y}}^{0}\left(\vec{a}, \gamma_{m_{x}, m_{y}}\right)$ and each single mirror can only be in the tilt state $\gamma^{-}$or $\gamma^{+}($as reasonable assumption for the steady state of a DMD). A Gaussian beam profile can still be approximated via varying $E_{m_{x}, m_{y}}^{0}$ over the different single mirrors, and as typically a reasonably sized array of mirrors is illuminated, the error introduced by this approximation is small. This provides us with the opportunity to solve the integral in eq. (7) over a single mirror for $\gamma^{-}$and $\gamma^{+}$, with the analytically known solution for $E_{\gamma^{ \pm}}^{\text {single mirror }}(\vec{a}, \vec{b}, w, \lambda)$ (see dmd.nb in the attached repository). Instead of calculating the field for each mirror individually, we can use the fields of a reference mirror for $\gamma^{-}$ and $\gamma^{+}$and apply the phase shift for the desired grid point $\left(m_{x}, m_{y}\right)$ shown in Figure 2c. This simplifies eq. (7) for the diffracted field (Figure 2e, middle) to:

$$
E_{D M D}\left(\vec{a}, \vec{b}, \gamma_{m_{x}, m_{y}}, w, m, N_{x}, N_{y}, \lambda\right)=\sum_{m_{x}=0, m_{y}=0}^{N_{x}-1, N_{y}-1} E_{m_{x}, m_{y}}^{0}\left(\vec{a}, \gamma_{m_{x}, m_{y}}\right) \cdot E^{\text {single mirror }}\left(\vec{a}, \vec{b}, \gamma_{m_{x}, m_{y}}^{ \pm}, w, \lambda\right) \cdot E_{m_{x}, m_{y}}^{\text {shift }}(\vec{a}, \vec{b}, m, \lambda)
$$

This approach is computationally much simpler, while only introducing a minor approximation. It was thus used for the design of our compact DMD-based SIM system [14].

\section{Grating approach:}

Additionally to the assumptions for the analytic phase shifting approach, we assume here that the field amplitude over the entire DMD is constant $\left(E_{m_{x}, m_{y}}^{0}\left(\vec{a}, \gamma_{m_{x}, m_{y}}\right)=1\right)$ and that all single mirrors are in the $\gamma^{-}$or $\gamma^{+}$state. The field $E_{\gamma^{ \pm}}^{\text {single mirror }}(\vec{a}, \vec{b}, w, \lambda)$ can be considered as envelope over the diffracted native DMD grid $E^{\text {grating }}\left(\vec{a}, \vec{b}, m, N_{x}, N_{y}, \lambda\right)$. This gives the opportunity to write eq. (7) as follows:

$$
E_{D M D}\left(\vec{a}, \vec{b}, \gamma^{ \pm}, w, m, N_{x}, N_{y}, \lambda\right)=E^{\text {single mirror }}\left(\vec{a}, \vec{b}, \gamma^{ \pm}, w, \lambda\right) \cdot E^{\text {grating }}\left(\vec{a}, \vec{b}, m, N_{x}, N_{y}, \lambda\right)
$$

To get the intensity $I_{D M D}$ we calculate and multiply both diffraction patterns $I_{\text {envelope }}$ and $I_{\text {grating }}$ (Figure 2d), very similar to the case of the Young double slit experiment:

$$
I_{D M D}=\left|E_{D M D}\right|^{2}=\left|E^{\text {single mirror }}\right|^{2} \cdot\left|E^{\text {grating }}\right|^{2}=I_{\text {envelope }} \cdot I_{\text {grating }}
$$

Of course, this approach is constrained to the simulation of a DMD without a pattern being displayed. However, if only the diffractive nature of the DMD itself is of concern, this offers a computationally very effective solution.

\section{Blaze condition approach:}

We assume that the incident beam is perpendicular to the tilting axes $\vec{n}$ of the single mirrors. This leads to $\vec{a}=\left(a_{x},-a_{x}, a_{z}\right)$ and $-\varphi_{a}=\vartheta_{a}$. To imagine it simply we rotate the DMD by $45^{\circ}$ (Figure $\left.3 a\right)$. To account for the rotation we switch to new angle coordinates (Figure $3 b$ ):

$$
\alpha=\arctan \left(\sqrt{2} \cdot \tan \left(\varphi_{a}\right)\right) \quad ; \quad \beta=\arctan \left(\sqrt{2} \cdot \tan \left(\varphi_{b}\right)\right)
$$

As new coordinate axes we define $\vec{k}=(1,-1,0)$. Along $\vec{k}$ we get a new lattice constant $m^{\prime}=\frac{1}{\sqrt{2}} m$. 
The pathlength is given by

$$
l=l_{a}-l_{b}=m^{\prime}(\sin (\alpha)-\sin (-\beta))
$$

For perfect constructive grating interference, the path difference $l$ must be an integer multiple of the wavelength $\lambda$ :

$$
n \lambda=m^{\prime}(\sin (\alpha)+\sin (\beta))
$$

If we have perfect constructive interference in the center of the envelope, the blaze condition is fulfilled. The center of the envelope can then be assumed as a reflection of the light incident on the surface of a single mirror and we define this as our diffraction angle $\beta=-\alpha+2 \gamma$. For this reflected envelope we can now calculate the corresponding diffraction order:

$$
n=\frac{m^{\prime}}{\lambda}(\sin (\alpha)+\sin (-\alpha+2 \gamma))
$$

If $n$ is an integer the blaze condition is fulfilled. We use $v$ to visualize this relationship:

$$
v=|\sin (n \pi)|=\left|\sin \left(\frac{\pi m^{\prime}}{\lambda}(\sin (\alpha)+\sin (-\alpha+2 \gamma))\right)\right|
$$

Here, $v=0$, whenever the blaze condition is fulfilled. This allows us to visualize the blaze condition for different wavelengths $\lambda$ and different incident angles $\alpha$ along the diagonal of the DMD (Figure 3b). While this approach is even more constrained, it has an important real-world application: It allows us to directly calculate incident and reflective angles when placing a DMD rotated by $45^{\circ}$ on an optical table, where collimated beams are expected to run parallel to the table's surface. Here, it provides a quick solution for the instrument design. A similar approach was

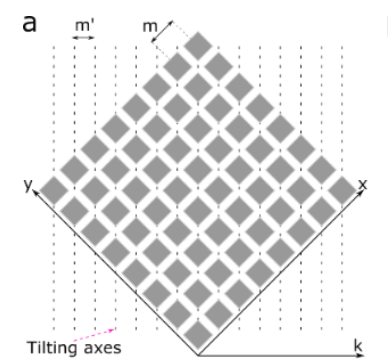

Figure 3: Blazed condition approach

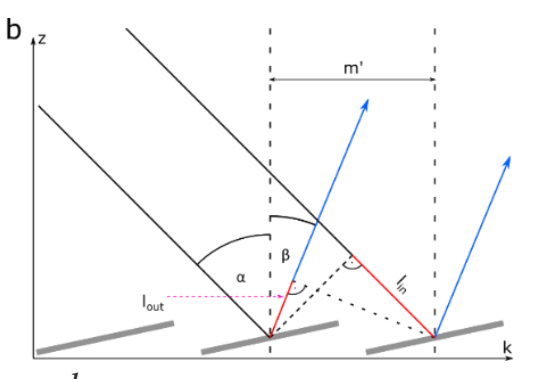

(a) Schematic illustration of a DMD rotated by $45^{\circ}$ around the $\mathrm{z}$-axis. Furthermore, the tilt axes of the micromirrors, the grid constants $m$ and $m^{\prime}$ and the coordinate axis $k$ are shown. (b) Graphical representation of the calculation of the phase shift for light, which is incident along the KZ-plane on a DMD. pursued by Li et al. [25]

\section{Results \& Discussion}

Each of the algorithms described above has certain advantages and disadvantages, which means that each algorithm has its own area of application. As shown in figure 1e, the ray-tracing approach, the analytic phase shifting approach and the lattice approach each deliver qualitatively the same results under identical boundary conditions. The positions of the individual diffraction orders are identical in the three algorithms. All diffraction images shown in this article were simulated with $m=7.56 \mu m$ micromirror pitch and $\gamma^{ \pm}= \pm 12^{\circ}$ as tilt angle, which corresponds to the dimensions of the $D L P ®$ LightCrafter ${ }^{\mathrm{TM}} 6500$ (Texas Instruments) [26] and some other DMDs. To obtain a clear visualization of the diffraction orders with large visible spots, the simulation is limited to an array of 50x50 mirrors being illuminated. Of course, when no or a different visualization is needed, the algorithms can be run for larger mirror arrays.

The ray tracing approach is most flexible because it requires the least simplifying approximations. It can be used to calculate the diffraction patterns for any DMD-pattern. In addition, the approach could be extended relatively easily for arbitrary exposure beam profiles and incoherent light by specifying a corresponding spatial distribution and wavelength distribution and/or phase distribution for the random rays. The main disadvantage of the ray tracing approach is that it is by far the slowest of the 


\begin{abstract}
presented algorithms. Therefore, this algorithm is especially suitable for the simulation of incoherent or partially coherent light. The analytical phase shifter has the only disadvantage that, when compared to the ray tracing approach, it cannot be readily modified for use with incoherent illumination, or if done so, it would lose its speed advantage. But the algorithm works much faster and is especially suitable for the calculation of diffraction images where the DMD is provided with a pattern (see Figure 4).
\end{abstract}

The grating approach is not able to simulate any patterns displayed on the DMD. It assumes that all mirrors are tilted in the same direction. Due to its high speed, however, the algorithm is very well suited for investigating the effects of different boundary conditions such as changes in wavelength and angle of incidence on the diffraction image generated natively by the structure of the micromirror array (Figure 5). It can be used to determine the distance between the center of the envelope and the brightest native grating order (Figure 5a). If this distance is $0^{\circ}$, the blaze condition (Littrow configuration) is fulfilled. For the use of coherent light in e.g. a SIM microscope, this is exactly what is desired, because then an isotropic envelope field and intensity distribution is present in the Fourier plane. This has the consequence that the disadvantages of the blazed grating effect of DMDs can be negated by exploiting the blaze condition. This is true for a range of angles of incidence which are shown in black in Figure 5b. These angles of incidence that fulfil the blaze condition then result in the angles of diffraction marked in Figure 5c.

If a DMD-based SIM microscope should require the use of multiple excitation wavelengths, then it is necessary that for each wavelength the blaze condition is fulfilled. A possible approach to solving this

\footnotetext{
Figure 5: Analysis of the displacement between the envelope and the brightest diffraction order of the DMD

The columns from left to right correspond to the wavelengths $488 \mathrm{~nm}, 532 \mathrm{~nm}$ and $561 \mathrm{~nm}$. Row (a) illustrates the displacement between the center of the envelope (gray, linear intensity representation) and the brightest diffraction order of the native DMD grating (cyan, green, yellow, logarithmic intensity representation) using red arrows. The field of view ranges from $-1^{\circ}$ to $7^{\circ}$ for $\varphi_{b}$ and $-7^{\circ}$ bis $1^{\circ}$ for $\vartheta_{b}$ at an angle of incidence of $\varphi_{a}=-\vartheta_{\mathrm{a}}=-21^{\circ}$. Row (b) visualizes the offset which can be determined from (a) for the angles of incidence $\varphi_{a}$ and $\vartheta_{a}$ ranging from $-60^{\circ}$ to $60^{\circ}$. The dark areas with a displacement close to $0^{\circ}$ indicate angles which fulfill a blaze condition and are well suited for illumination with coherent light. The cyan dotted lines between (a) and (b) indicate an angle of incidence of $\varphi_{a}=-\vartheta_{\mathrm{a}}=-21^{\circ}$ for the diffraction images seen in (a). Row (c) shows the areas in the diffraction space of $\varphi_{b}$ and $\vartheta_{b}$ from $-80^{\circ}$ to $80^{\circ}$, each, in which the displacement between the center of the envelope and the brightest diffraction order of the DMD is not more than $0.1^{\circ}$. Therefore, angles of incidence in a range of $-60^{\circ}$ to $60^{\circ}$ used in (b) were used for $\varphi_{a}$ and $\vartheta_{a}$. The red boxes mark the diffraction space shown in (a). The white dotted lines in (b) and (c) correspond to the diagonal angles of incidence that are considered in the blazed condition approach.
}
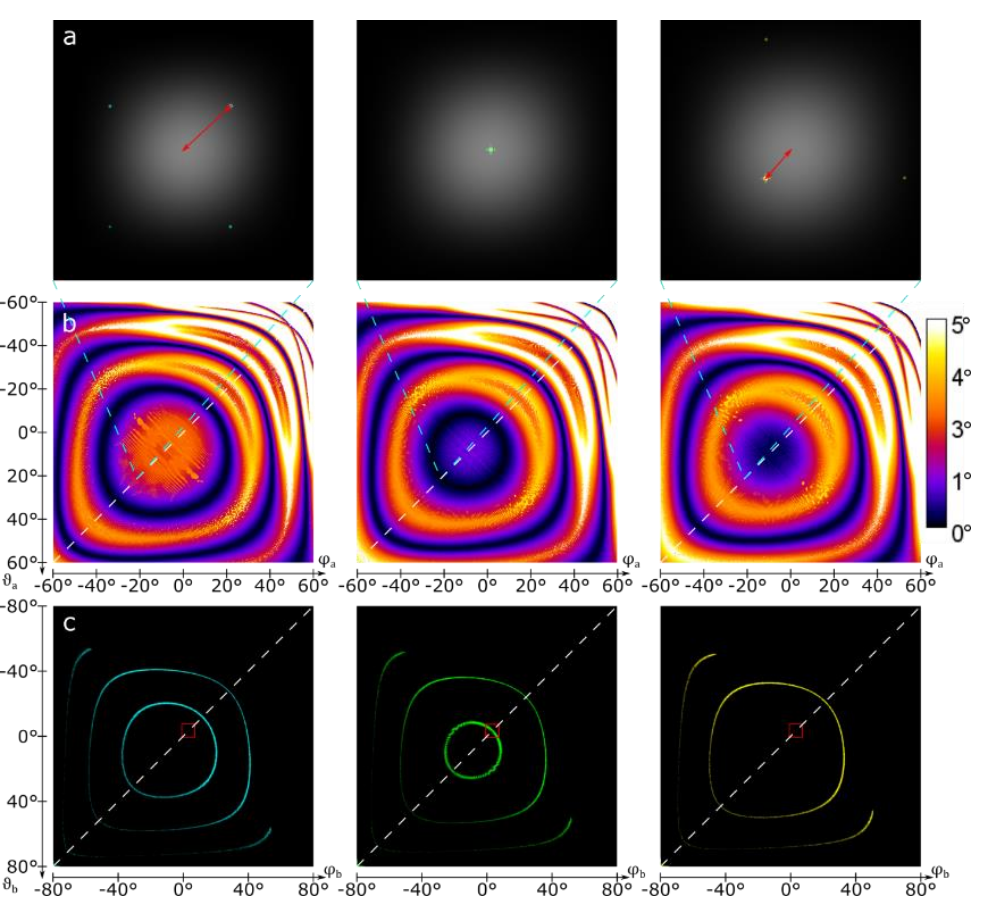
problem is to use the $\gamma^{-}$position of the micromirrors for one wavelength and the $\gamma^{+}$position for the other wavelength. As shown for example for $488 \mathrm{~nm}$ or $638 \mathrm{~nm}$ in combintion with $561 \mathrm{~nm}$ in Figure $7 \mathrm{a}$, there are two configurations in which

the blaze condition is fulfilled for both wavelengths. This configuration is, however, very difficult to achieve experimentally (as multiple independent tip/tilt axis have to be precisely aligned) and it is also very susceptible to the slightest change in angle of the micromirrors (we found e.g. $0.3^{\circ}$ in [14]). Therefore, we propose to limit the angle of incidence and thus also the outgoing angle for the optical axis of the DMD-SIM microscope to the diagonal perpendicular to the tilting axis of the micromirrors. This corresponds to a rotation of the DMD of $45^{\circ}$ around the $\mathrm{z}$-axis (Figure 3). This limits the possible angles of incidence as well as the angles of diffraction for the optical

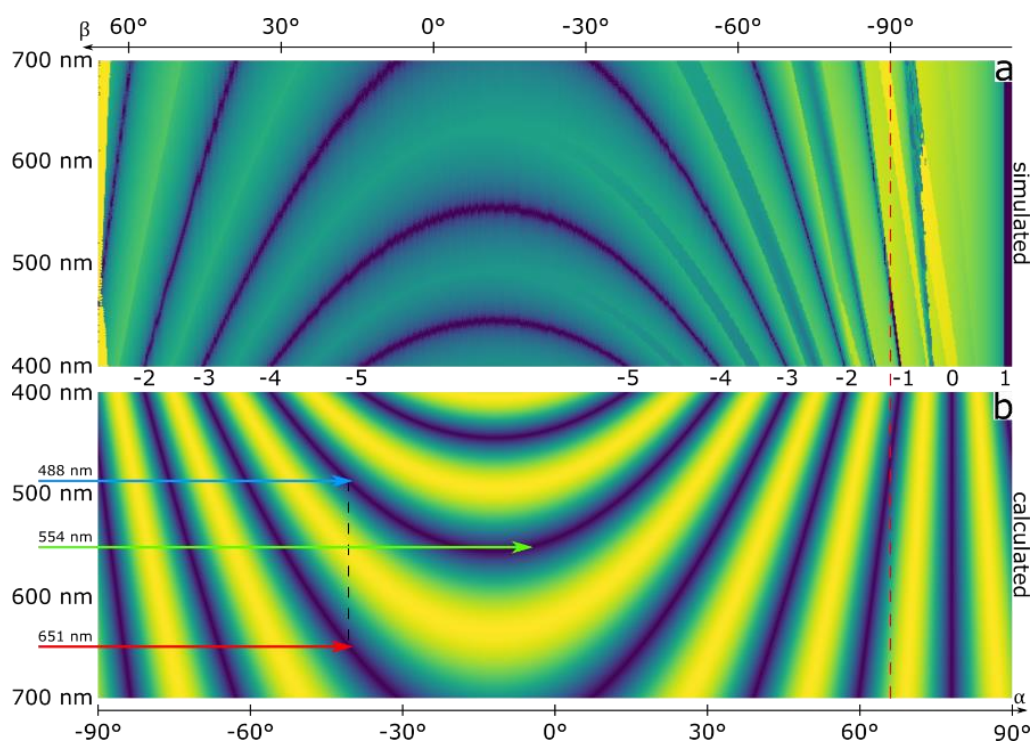

Figure 6: Blaze condition along the diagonals shown in Figure $3 b$

Visualization of the blaze condition along the diagonals shown in Figure $3 \mathrm{~b}$ for the visible spectrum. In the dark areas the blaze condition can be considered fulfilled. For orientation, the wavelength range is shown on the left-hand side. The angles of incidence and diffraction are shown at the top and the bottom of the graph. The vertical red dashed line marks the diffraction angle of $90^{\circ}$. Angles above $90^{\circ}$ are irrelevant for practical applications. (a) The blaze condition is measured by the distance between the center of the envelope and the brightest diffraction order of the DMD, which was simulated by the grating approach. The measured distances are shown as logarithmic intensity distribution. (b) Visualization $v=|\sin (n \pi)|$ of the blaze condition using the blazed-grating approach. The integer diffraction orders belonging to the blaze conditions are shown in the middle between (a) and (b). The blue, red and green arrows point to an exemplary three-color combination for a DMD-SIM microscope with $488 \mathrm{~nm}, 651 \mathrm{~nm}$ and $554 \mathrm{~nm}$. The black vertical dotted line is intended to illustrate that $488 \mathrm{~nm}$ and $651 \mathrm{~nm}$ fall on the DMD at the same angle of incidence.

axis to the white dotted diagonal in fig $5 \mathrm{~b}$. This reduces the number of degrees of freedom of the incident laser beam $\left(\varphi_{a}, \vartheta_{a}\right) \rightarrow \alpha$ and the optical axis $\left(\varphi_{b}, \vartheta_{b}\right) \rightarrow \beta$ from two to one each, as described in eq. (14). The experimental implementation thus becomes much easier, because the exposure path of the microscope can be set up parallel to the optical table, as usual. Now, however, the wavelengths must be chosen skilfully. For a more precise analysis of the blaze condition along the diagonal, both the grating approach and the blazed condition approach are suitable.

Both approaches show qualitatively the same results, therefore it is sufficient to refer to the blaze condition approach in the following. It is possible to use two wavelengths $\lambda_{1}$ and $\lambda_{2}$ with the same angle of incidence $\alpha_{1,2}$ and diffraction $\beta$ with the same tilt state $\gamma^{-}=-12^{\circ}$. Starting from eq. (16) it follows that

$$
n_{1} \lambda_{1}=n_{2} \lambda_{2}
$$

As integer diffraction orders $n_{1}=-4$ and $n_{2}=-3$ are suitable for the visible range. If we pretend $\lambda_{1}=$ $488 \mathrm{~nm}$ we obtain:

$$
\lambda_{2}=\frac{n_{1}}{n_{2}} \cdot \lambda_{1}=650.67 \mathrm{~nm} \quad \text { with } \quad \alpha_{1,2}=-40.6^{\circ} \quad \text { and } \quad \beta=16.6^{\circ}
$$

If a third excitation color is supposed to utilize the other tilt state $\gamma^{+}=12^{\circ}$, we get $\alpha_{3}=-\beta+2 \gamma^{+}=7,4^{\circ}$ as incidence angle and must assume $n_{3}=4$ to be in the visible range, which then limits the wavelength to:

$$
\lambda_{3}=\frac{m^{\prime}}{n_{3}}\left(\sin \left(\alpha_{3}\right)+\sin (\beta)\right)=553.93 \mathrm{~nm}
$$


This combination of these three wavelengths, arranged at $\left(-40.6^{\circ}, 488 \mathrm{~nm}\right), \quad\left(-40.6^{\circ}, 651 \mathrm{~nm}\right)$ and $\left(7.4^{\circ}, 554 \mathrm{~nm}\right)$ is indicated in Figure 6 as a possible configuration for a three color DMD-SIM microscope. Given that these wavelengths are close to the typical combinations of blue (488nm), yellow (561nm, 568nm) and red (635nm to $650 \mathrm{~nm}$ ) laser excitation sources used in fluorescence microscopy, and that suitable diode laser sources (even offering temperature-based finetuning of their emission wavelength) are readily available, this combination should make for a surprisingly capable three-color DMD-based microscope.

Further possible configurations are e.g. $473 \mathrm{~nm}, 631 \mathrm{~nm}$ and $551 \mathrm{~nm}$ or $491 \mathrm{~nm}, 655 \mathrm{~nm}$ and $555 \mathrm{~nm}$. It is

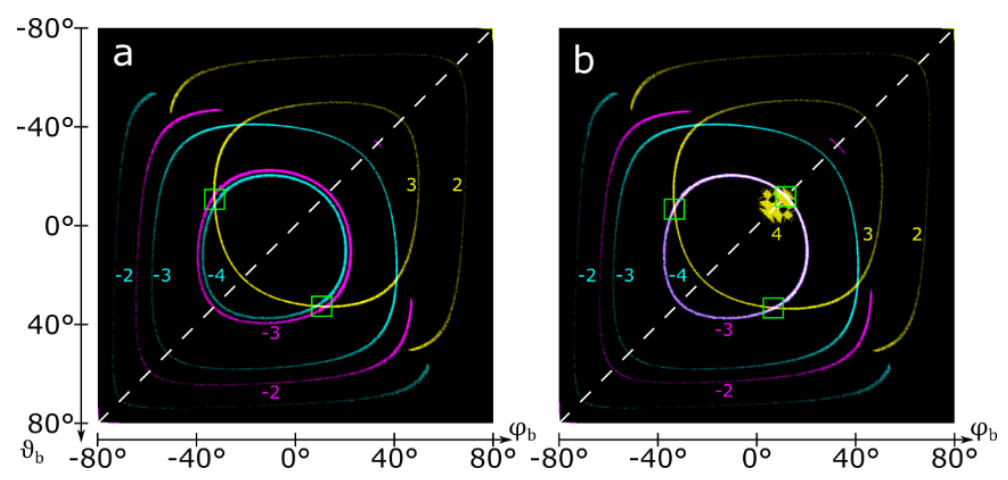

Figure 7: Multi color diffraction angles with blaze condition

Areas in the diffraction space for $\varphi_{b}$ and $\vartheta_{b}$ of $-80^{\circ}$ to $80^{\circ}$, each, in which the displacement between the center of the envelope and the brightest diffraction order of the DMD is not more than $0.1^{\circ}$. Therefore, angles of incidence in a range of $-60^{\circ}$ to $60^{\circ}$ were used for $\varphi_{a}$ and $\vartheta_{a}$. The white dotted lines correspond to the diagonal angles of incidence that are considered in the blazed condition approach. The colored numbers near the ring structures represent the corresponding diffraction orders. (a) Typical wavelengths $488 \mathrm{~nm}$ (displayed in cyan) \& $638 \mathrm{~nm}$ (displayed in magenta) with $\gamma^{-}=-12^{\circ}$ and $561 \mathrm{~nm}$ (displayed in yellow) with $\gamma^{+}=12^{\circ}$ are not well suited for multicolor applications. The green boxes mark possible configurations for two color configurations of $488 \mathrm{~nm} \& 561 \mathrm{~nm}$ or $638 \mathrm{~nm}$ \& $561 \mathrm{~nm}$. (b) Optimized multicolor wavelengths $488 \mathrm{~nm}$ (cyan) \& $651 \mathrm{~nm}$ (magenta) with $\gamma^{-}=-12^{\circ}$ and $554 \mathrm{~nm}$ (yellow) with $\gamma^{+}=12^{\circ}$. The green boxes mark possible configurations for three color configurations.

important to note here that $\lambda_{3}$ depends very much on the tilt angles of the micromirrors, and thus might have to be adjusted based on the parameters of the specific DMD device. For $\lambda_{1}$ and $\lambda_{2}$ the tilt angle is of less importance. Looking at the diffraction space in which the blaze condition is fulfilled for typical wavelengths (Figure 7a), it is noticeable that, using the same tilt state, no matches are found. The ringlike structures in Figure 7 represent the different diffraction orders. Their size depends on wavelength and diffraction order and for clarity they are shown in different colors. As mentioned earlier, using the other tilt state results in matches, which are not on the diagonal and therefore not suitable for experiments. But if we choose the wavelengths carefully for multicolor applications (fig 7b), the circular structure of the -4 th and -3rd diffraction order of $488 \mathrm{~nm}$ and $651 \mathrm{~nm}$ using the same tilt state match exactly. If we use the other tilt state for $554 \mathrm{~nm}$, there is, besides the matches outside the diagonal, another one on the diagonal, which is formed by a collapsing/emerging circular structure of the 4th diffraction order. This configuration, already described above, is therefore ideal for a three-color DMDSIM microscope.

\section{Conclusions}

The framework and the software tools provided here provide a rather universal set of simulations tools for experimentalists planning to use DMDs as spatial light modulators with coherent light sources in SIM microscopes or similar applications. Compared to ferro-electric light modulators (FLCoS-SLM), DMDs are faster and more cost efficient, and they allow for a more compact design due to the smaller

Table 1: Comparison of all discussed approaches

\begin{tabular}{|l|l|l|l|l|}
\hline Approach & Ray tracing & $\begin{array}{l}\text { Analytic phase } \\
\text { shifting }\end{array}$ & Grating & Blaze condition \\
\hline Capable of patterns & + Yes & + Yes & - No & - No \\
\hline $\begin{array}{l}\text { Speed } \\
\text { (Time CPU/GPU) }\end{array}$ & $\begin{array}{l}-- \text { Very slow } \\
(84 \mathrm{~min} /----)\end{array}$ & $\begin{array}{l}- \text { Slow } \\
(7.5 \mathrm{~min} / 3 \mathrm{sec})\end{array}$ & $\begin{array}{l}\text { + Fast } \\
(3.2 \mathrm{sec} / 1.2 \mathrm{sec})\end{array}$ & ++ Instant \\
\hline Use case & $\begin{array}{l}\text { Incoherent } \\
\text { light }\end{array}$ & $\begin{array}{l}\text { Specific DMD- } \\
\text { patterns }\end{array}$ & $\begin{array}{l}\text { Native pattern } \\
\text { under different } \\
\text { conditions }\end{array}$ & $\begin{array}{l}\text { Analysis of the } \\
\text { diagonal }\end{array}$ \\
\hline
\end{tabular}

This table gives a compact summary of the discussed approaches. The runtimes for the approaches were measured with $50 \times 50$ mirrors at a resolution of $1500 \times 1500$ pixels in diffraction space on an Intel Core i5 4690 4x 3.50GHz with a NVIDIA GeForce GTX 1060 6GB. The ray tracing approach was measured with 100000 rays. The runtimes can vary significantly depending on the approach, the number of mirrors, the resolution and the computer used. 
micromirror/pixel pitch. Also, the use of FLCoS-SLMs requires the implementation of a much more complicated timing scheme because of the limited duration during which a specific pattern can be projected by these devices. DMDs have no such constraints and can switch at speeds up to an order of magnitude faster than FLCoS-SLMs. We have developed four different approaches to simulate coherent light diffracted and reflected by a DMD (table 1). The results provide practical design suggestions for the circumventing the undesired blazed-grating effect of DMDs. Therefore, it is now possible to design a multicolor DMD-based SIM microscope with simple opto-mechanics which can fully utilize all of the advantages of a DMD mentioned before. We are currently building a two-color DMD SIM microscope and plan to add a third color to it in its final implementation.

The results shown in this article can easily be reproduced with the ImageJ/Fiji plugin which we provide as an open access download. The plugin contains all approaches and algorithms presented in this article, which can be adapted to different system conditions. For a more detailed insight into the plugin and some mathematical additions we recommend a look at the supplementary material.

Following the spirit of open science, we provide all source code and raw data for the results presented in this manuscript to the scientific community. All code is openly accessible under GPLv2 (or later) license and can be found in online repositories under github.com/fairSIM and github.com/biophotonicsbielefeld.

\section{Additional Information}

\section{Data Accessibility}

A software package to simulate with the introduced algorithms the diffraction patterns of DMDs and the introduced further analysis depending on the angle of incidence, light wavelength and DMD pattern has been created. The software is written in Java to work as a plugin to scientific image processing package ImageJ/FIJI $[27,28]$. and provides GPU acceleration through the jCuda framework [29]. The source code is available under an open-source (GPLv3) licence in an online repository: github.com/biophotonics-bielefeld/coherent-dmd-sim-simulator.

\section{Authors' Contributions}

ML wrote the DMD simulation software, developed the analytic phase shifting approach, the grating approach, the blazed condition approach, set up electronic and control software of the DMD-SIM microscope and drafted the manuscript. HS developed the ray tracing approach and crosschecked math and simulations. AS built the DMD-SIM microscope, performed the SIM measurements and processed the SIM reconstructions. TH and JSaE supervised the project and helped write the manuscript. MM envisioned the project and wrote the manuscript. All authors read and approved the manuscript.

\section{Competing Interests}

The authors declare that they have no competing interests.

\section{Funding Statement}

We acknowledge funding by the Deutsche Forschungsgemeinschaft (DFG, German Science Foundation) - project number 415832635 . This project has also received funding from the European Union's Horizon 2020 research and innovation programme under the Marie Skłodowska-Curie grant agreements No. 752080. ML was supported by the Protestant Hospital of Bethel Foundation.

\section{References}

1. Maurer C, Jesacher A, Bernet S, Ritsch-Marte M. 2011 What spatial light modulators can do for optical microscopy. Laser Photonics Rev. 5, 81-101. (doi:10.1002/lpor.200900047)

2. Fulwyler M, Hanley QS, Schnetter C, Young IT, Jares-Erijman EA, Arndt-Jovin DJ, Jovin TM. 2005 Selective photoreactions in a programmable array microscope (PAM): Photoinitiated polymerization, photodecaging, and photochromic conversion. Cytometry A 67A, 68-75. (doi:10.1002/cyto.a.20174) 
bioRxiv preprint doi: https://doi.org/10.1101/2020.10.02.323527; this version posted October 3, 2020. The copyright holder for this preprint (which was not certified by peer review) is the author/funder. All rights reserved. No reuse allowed without permission.

3. Heintzmann R, Cremer CG. 1999 Laterally modulated excitation microscopy: improvement of resolution by using a diffraction grating. In (eds IJ Bigio, H Schneckenburger, J Slavik, K Svanberg, PM Viallet), pp. 185-196. Stockholm, Sweden: Proc. SPIE. (doi:10.1117/12.336833)

4. Gustafsson MGL. 2000 Surpassing the lateral resolution limit by a factor of two using structured illumination microscopy. J. Microsc. 198, 82-87. (doi:10.1046/j.1365-2818.2000.00710.x)

5. Gustafsson MGL, Shao L, Carlton PM, Wang CJR, Golubovskaya IN, Cande WZ, Agard DA, Sedat JW. 2008 ThreeDimensional Resolution Doubling in Wide-Field Fluorescence Microscopy by Structured Illumination. Biophys. J. 94, 49574970. (doi:10.1529/biophysj.107.120345)

6. Lu-Walther H-W, Kielhorn M, Förster R, Jost A, Wicker K, Heintzmann R. 2015 fastSIM: a practical implementation of fast structured illumination microscopy. Methods Appl. Fluoresc. 3, 014001. (doi:10.1088/2050-6120/3/1/014001)

7. Chang B-J, Chou L-J, Chang Y-C, Chiang S-Y. 2009 Isotropic image in structured illumination microscopy patterned with a spatial light modulator. Opt. Express 17, 14710. (doi:10.1364/OE.17.014710)

8. Förster R, Lu-Walther H-W, Jost A, Kielhorn M, Wicker K, Heintzmann R. 2014 Simple structured illumination microscope setup with high acquisition speed by using a spatial light modulator. Opt. Express 22, 20663. (doi:10.1364/OE.22.020663)

9. Wu Y, Shroff H. 2018 Faster, sharper, and deeper: structured illumination microscopy for biological imaging. Nat. Methods 15, 1011-1019. (doi:10.1038/s41592-018-0211-z)

10. Demmerle J et al. 2017 Strategic and practical guidelines for successful structured illumination microscopy. Nat. Protoc. 12, 988-1010. (doi:10.1038/nprot.2017.019)

11. Schermelleh L, Ferrand A, Huser T, Eggeling C, Sauer M, Biehlmaier O, Drummen GPC. 2019 Super-resolution microscopy demystified. Nat. Cell Biol. 21, 72-84. (doi:10.1038/s41556-018-0251-8)

12. Li D et al. 2015 Extended-resolution structured illumination imaging of endocytic and cytoskeletal dynamics. Science 349, 3500. (doi:10.1126/science.aab3500)

13. Markwirth A, Lachetta M, Mönkemöller V, Heintzmann R, Hübner W, Huser T, Müller M. 2019 Video-rate multi-color structured illumination microscopy with simultaneous real-time reconstruction. Nat. Commun. 10, 4315. (doi:10.1038/s41467-019-12165-x)

14. Sandmeyer A, Lachetta M, Sandmeyer H, Hübner W, Huser T, Müller M. 2019 DMD-based super-resolution structured illumination microscopy visualizes live cell dynamics at high speed and low cost. (doi:10.1101/797670)

15. Dan D et al. 2013 DMD-based LED-illumination Super-resolution and optical sectioning microscopy. Sci. Rep. 3. (doi:10.1038/srep01116)

16. Descloux A et al. 2019 High speed multi-plane super-resolution structured illumination microscopy of living cells using an image-splitting prism. (doi:10.1101/773440)

17. Reymond L, Ziegler J, Knapp C, Wang F-C, Huser T, Ruprecht V, Wieser S. 2019 SIMPLE: Structured illumination based point localization estimator with enhanced precision. Opt. Express 27, 24578. (doi:10.1364/OE.27.024578)

18. Chakrova N, Canton AS, Danelon C, Stallinga S, Rieger B. 2016 Adaptive illumination reduces photobleaching in structured illumination microscopy. Biomed. Opt. Express 7, 4263. (doi:10.1364/BOE.7.004263)

19. Shin S, Kim D, Kim K, Park Y. 2018 Super-resolution three-dimensional fluorescence and optical diffraction tomography of live cells using structured illumination generated by a digital micromirror device. Sci. Rep. 8, 9183. (doi:10.1038/s41598-01827399-w)

20. Li M et al. 2020 Structured illumination microscopy using digital micro-mirror device and coherent light source. Appl. Phys. Lett. 116, 233702. (doi:10.1063/5.0008264)

21. Lei M, Zhou X, Dan D, Qian J, Yao B. 2016 Fast DMD based super-resolution structured illumination microscopy. In Frontiers in Optics 2016 (2016), paper FF3A.5, p. FF3A.5. Optical Society of America. (doi:10.1364/FIO.2016.FF3A.5)

22. Brown PT, Kruithoff R, Seedorf GJ, Shepherd DP. 2020 Multicolor structured illumination microscopy and quantitative control of coherent light with a digital mirror device. (doi:10.1101/2020.07.27.223941)

23. TI Instruments. 2008 Using Lasers with DLP® DMD technology.

24. Chen X, Yan B, Song F, Wang Y, Xiao F, Alameh K. 2012 Diffraction of digital micromirror device gratings and its effect on properties of tunable fiber lasers. Appl. Opt. 51, 7214-7220. (doi:10.1364/AO.51.007214)

25. Li M et al. 2020 Structured illumination microscopy using digital micro-mirror device and coherent light source. Appl. Phys. Lett. 116, 233702. (doi:10.1063/5.0008264)

26. Texas Instruments EA. 2016 DLP6500 0.65 1080p MVSP Type A DMD datasheet.

27. Schneider CA, Rasband WS, Eliceiri KW. 2012 NIH Image to ImageJ: 25 years of image analysis. Nat. Methods 9, 671-675. (doi:10.1038/nmeth.2089)

28. Schindelin J et al. 2012 Fiji: an open-source platform for biological-image analysis. Nat. Methods 9, 676-682. (doi:10.1038/nmeth.2019)

29. Yan Y, Grossman M, Sarkar V. 2009 JCUDA: A Programmer-Friendly Interface for Accelerating Java Programs with CUDA. In Euro-Par 2009 Parallel Processing (eds H Sips, D Epema, H-X Lin), pp. 887-899. Berlin, Heidelberg: Springer Berlin Heidelberg. (doi:10.1007/978-3-642-03869-3_82)

30. Kř́žek P, Lukeš T, Ovesný M, Fliegel K, Hagen GM. 2015 SIMToolbox: a MATLAB toolbox for structured illumination fluorescence microscopy. Bioinformatics, btv576. (doi:10.1093/bioinformatics/btv576)

31. Lukeš T, Kř́žek P, Švindrych Z, Benda J, Ovesný M, Fliegel K, Klíma M, Hagen GM. 2014 Three-dimensional superresolution structured illumination microscopy with maximum a posteriori probability image estimation. Opt. Express 22, 29805. (doi:10.1364/OE.22.029805)

32. Pospis $`$ il J, Spendier K, Hagen GM. In press. Imaging tissues and cells beyond the diffraction limit with structured illumination microscopy and Bayesian image reconstruction. GigaScience 8, 12. 